\title{
UNIVERSITY \\ OF OTTAWA \\ Faculty of Medicine
}

Graduate Training Program in Neurology. Combining the neurology services of the Ottawa Civic Hospital and the Ottawa General Hospital, a four-year integrated training program, fully accredited by the Royal College of Physicians and Surgeons of Canada, is offered. Trainees may enter for the entire program or at various points in it. The program contains considerable flexibility to recognize the interests and past experience of the trainee. Options are available for rotation through neuropathology, neuroradiology, neuro-ophthalmology, neuropsychology, psychiatry, EEGclinical neurophysiology, or basic neurological sciences. The latter includes neuroanatomy, neurophysiology, neurochemistry, and neuropharmacology. Registration for University of Ottawa graduate degrees may be arranged. Approximately 25 University staff members are involved in the training program. Applicants should apply by November 30th, for the following year. Under special circumstances, or if positions are available, applications will, however, be considered at other times. Applications and requests for more details should be sent directly to Dr. E. A. Atack, Program Director, Postgraduate Training Program in Neurology, University of Ottawa, 1081 Carling Ave. No. 303, Ottawa, Ontario K1Y 4G2. 
MOVING?

\section{PLEASE NOTIFY US OF YOUR CHANGE OF ADDRESS IN ADVANCE.}

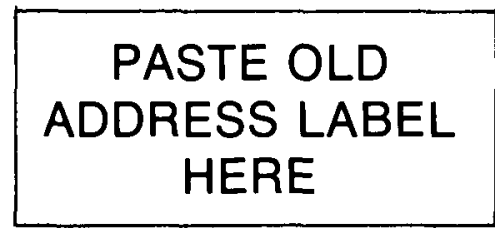

NEW ADDRESS:

NAME:

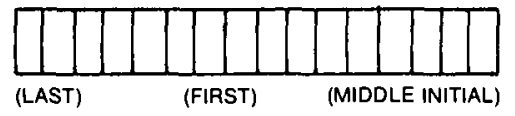

STREET ADDRESS:

CITY:

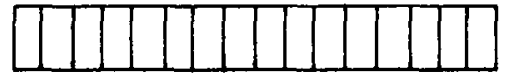

PROVINCE/STATE:

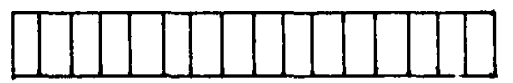

\section{COUNTRY:}

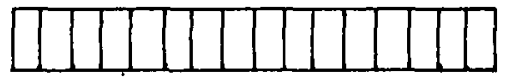

\section{POSTAL/ZIP CODE:}

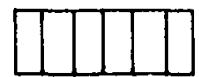

\section{MAIL TO:}

C.J.N.S.

1516 - 233 Kennedy St.

Winnipeg, Canada R3C 3J5

\section{In epilepsy}

Tegretol

provides control of seizures and alleviation of personality disorders

Roterences

Livingston, S. F.: Comprehensive Management of Epilepsy in Infancy, Childhood and Adolescence, Charles C. Thomas, 1972

2 Rodin, E. A., Rim, G. S., and Rennick, P.: Abstract from Program of the American Epilepsy Society Annual Meeting (Dec. 6) 1973, N.Y.

(Tegretol) in Epilepsy Nine Year Follow-up Study with Special Emphasis (March) 1974.

Brief Prescribing Information

Tegretol' $200 \mathrm{mg}$

Anticonvulsan

Properties
Tegretol has a proven anticonvulsant effect. In addition, Tegretol also has a distinct psychotropic effect, improving with asd and Tha trigeminal neuralgia, usually within $24-48$ hours. Indications

1 Epilepsy

Temporal lobe (psychomotor) epilepsy, and as an adjunct in secondary epilepsy or partial epilepsy with complex symptoms or secondarily generalized seizures.

2 Neuralgia

Trigeminal neuralgia (tic douloureux), glossopharyngeal neuralgia.

Dosage

A gradual increasing schedule is recommended with adjustment to suit the needs of the individual. When Tegretol is added to, or substituted for, existing ant convulsant therapy, the dosage

should be

Initially $y_{2}-1$ tablet $(100 \mathrm{mg}-200 \mathrm{mg})$ twice daily increas ing over a period of $4-6$ days until optimal control is achieved (usually with 3 tablets daily).

Trigeminal Neuralgia

Initially $-200 \mathrm{mg}$ daily in divided doses of $100 \mathrm{mg}$ ( $/ y_{2}$ tablet), increasing by $200 \mathrm{mg}$ ( 1 tablet) daily until pain relief is obtained. Dosage in excess of $1200 \mathrm{mg}$ (6 tablets)

daily is not recommenced.

All patients should be maintained on the minimum effective dose.

Adverse Reactions

Most frequently reported are: drowsiness, disturbances of accommodation, vertigo, dizziness and gastrointestinal disturbances. They usually occur only during initial phase startina and can be mized, on the circulatory system have been reported The mary and adverse reactions which may require discontinuation of therapy are the haematological including blood dyscra. sias, the hepatic including jaundice, the dermatological, the neurological, the cardiovascular, the genito-urinary, the digestive, and the ocular. Miscellaneous including fever and chills, lymphadenopathy aching joints and muscles, leg cramps and conjunctivitis.

Precautions

Careful clinical and laboratory supervision should be instituted prior to and maintained throughout treatment. Caution should be observed while treating patients with increased ocular pressure or uninary reten patients wh a history of cor uriary ary of agitation and confusion in the elderly or activating a latent psychosis.

Contraindications

Concomitant use of monoamine oxidase inhibitors (two weeks should elapse before Tegretol is prescribed for patients who have received MAOI drugs), first trimester of pregnancy, nursing mothers, patients with a history of hepatic disease or serious blood disorder, or known sensitivity to any tricyclic compound. Tegretol should not be given to women of child-bearing potential unless, in the opinion of the physician, the expected benefits to the patient outweigh the possible risk to the foetus.

Warnings

Although reported infrequently, serious adverse effects have been observed during the use of Tegrotol. Agranulocytosis and aplastic anemia have occurred in a few instances with a fatal outcome. Leucopenia, thrombo. cytopenia and hepatocellular and cholestatic jaundice have also been reported. It is, therefore, important that Tegretol should be used carefully and close clinical and frequent laboratory supervision should be maintained throughout treatment in order to detect as early as possible signs and symptoms of a possible blood dyscrasia.

Tyerdosage

No specific antidote.

Availability

Each round, white, single scored tablet with seal contains: carbamazepine $200 \mathrm{mg}$, available in

bottles of 50 and 500 .

Full information is available on request.

\section{Geigy}



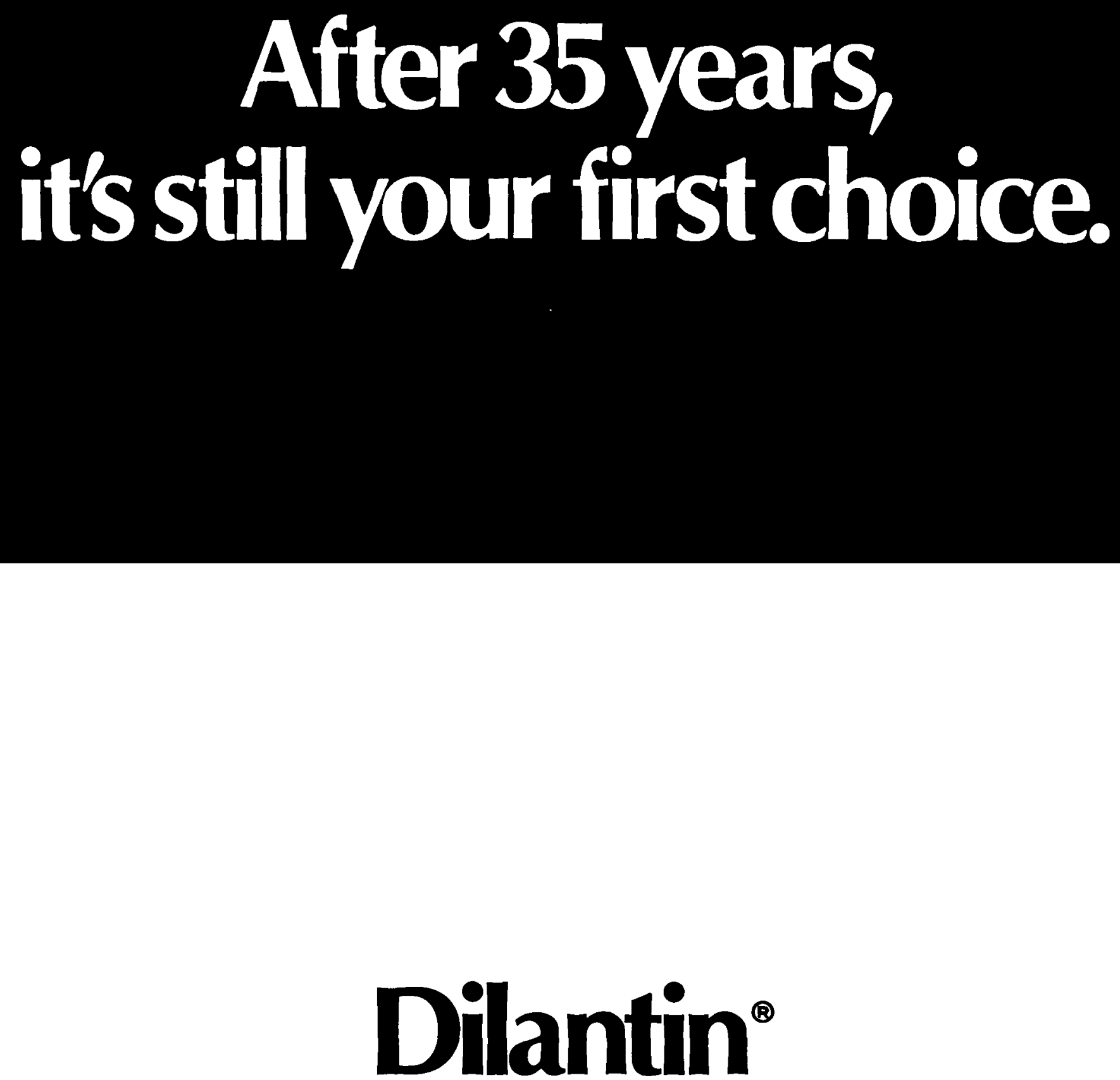

(diphenylhydantoin)

Little wonder you've continually backed the complete Parke-Davis line of anticonvulsants: Dilantin ${ }^{\oplus}$, Zarontin ${ }^{\circledR}$ (ethosuximide), - Dilantin ${ }^{\oplus}$ with phenobarbital, ${ }^{\oplus}$ Phelantin ${ }^{*}$

(Dilantin, phenobarbital, and methamphetamine hydrochloride). Celontin ${ }^{(8)}$

(methsuximide), and Milontin ${ }^{\circledast}$ (phensuximide).

Detailed information available upon request.

\section{PARKE-DAVIS}

Parke, Davis \& Company, Ltd., Don Mills, Ontario. 


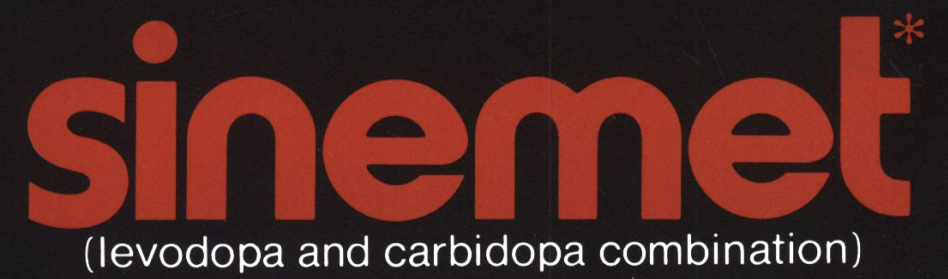

\section{a most significant \\ advance in the treatment of Parkinson's syndrome}

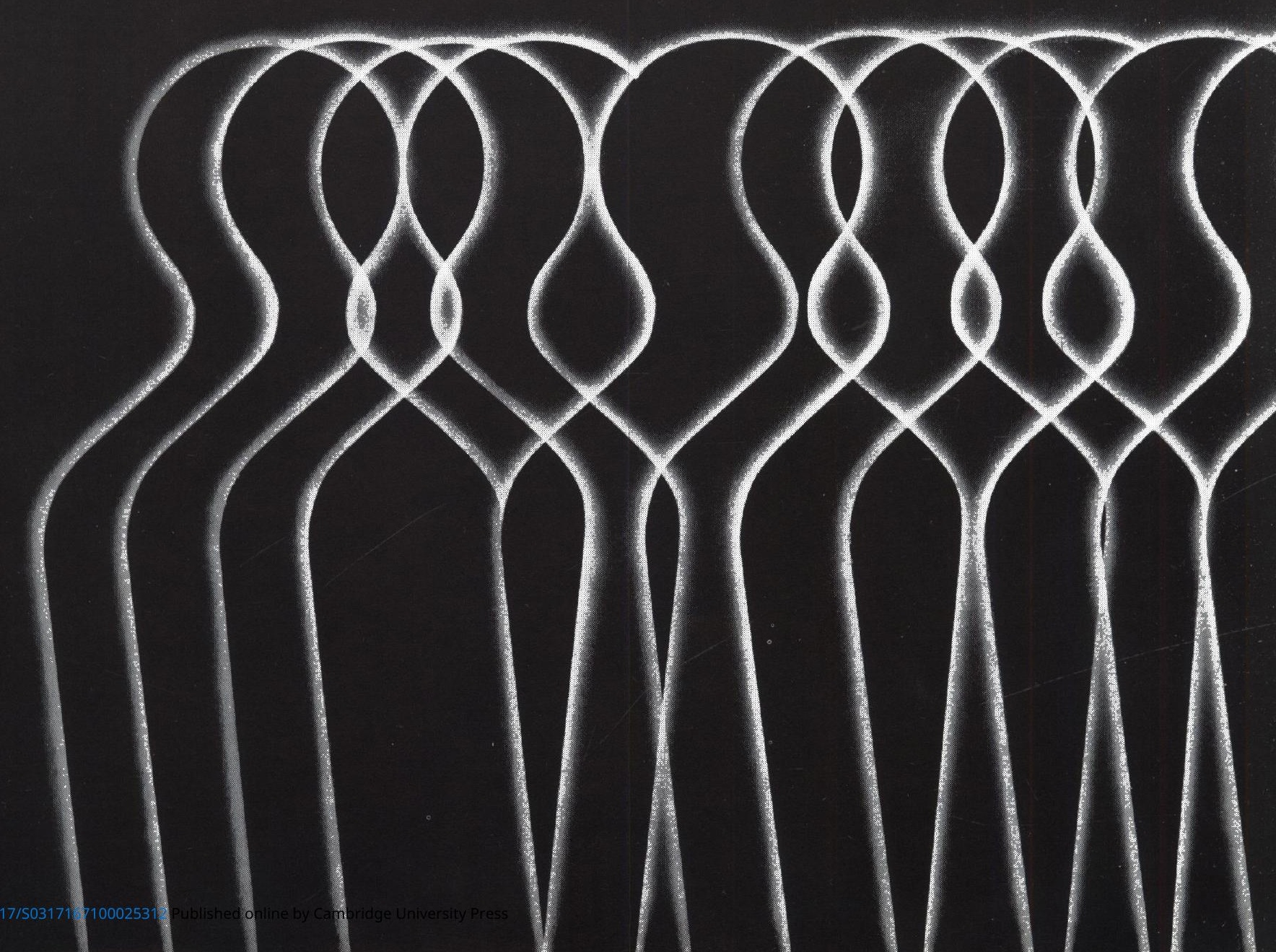




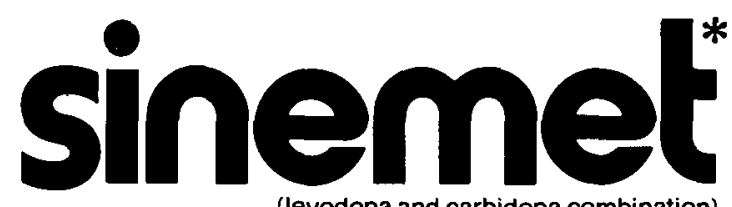

(levodopa and carbidopa combination)

\section{INDICATIONS}

Treatment of Parkinson's syndrome with exception of drug induced parkinsonism.

\section{CONTRAINDICATIONS}

When a sympathomimetic amine is contraindicated; with monoamine oxidase inhibitors, which should be discontinued two weeks prior to -starting SINEMET*: in uncompensated cardiovascular, endocrine, hematologic hepatic, pulmonary or renal disease; in narrowangle glaucoma; in patients with suspicious undiagnosed skin lesions or a history of melanoma.

\section{WARINGS}

When given to patients receiving levodopa alone, discontinue levodopa at least 12 hours before initiating SINEMET * at a dosage that provides approximately $20 \%$ of previous levodopa.

Not recommended in drug-induced extrapyramidal reactions; contraindicated in management of intention tremor and Huntington's chorea.

Levodopa related central effects such as involuntary movements may occur at lower dosages and sooner, and the 'on and off phenomenon may appear earlier with combination therapy.

Monitor carefully all patients for the development of mental changes, depression with suicidal tendencies, or other serious antisocial behaviour.

Cardiac function should be monitored conti nuously during period of initial dosage adjustment in patients with arrhythmias.

Safety of SINEMET * in patients under 18 years of age not established.

Pregnancy and lactation: In women of childbearing potential, weigh benefits against risks. Should not be given to nursing mothers. Effects on human pregnancy and lactation unknown.

\section{PRECAUTIONS}

General: Periodic evaluations of hepatic hematopoietic, cardiovascular and rena function recommended in extended therapy. Treat patients with history of convulsions cautiously. Physical Activity: Advise patients improved on SINEMET* to increase physical activities gradually, with caution consistent with other medical considerations. In Glaucoma: May be given cautiously to patients with wide angle glaucoma. provided intraocular pressure is well controlled and can be carefully monitored during therapy. With Antihypertensive Therapy: As symptomatic postural hypotension has been reported occasionally give cautiously to patients on antihypertensive drugs, checking carefully for changes in pulse rate and blood pressure. Dosage adjustment of antihypertensive drug may be required. With Psychoactive Drugs: If concomitant administration is necessary, administer psychoactive drugs with great caution and observe patients for unusual adverse reactions. With Anes thetics: Discontinue SINEMET* the night before general anesthesia and reinstitute as soon as patient can take medication orally.

\section{ADVERSE REACTIONS}

Most Common: Abnormal Involuntary Move ments-usually diminished by dosage reduction-choreiform, dystonic and other involuntary movements. Muscle twitching and blepharospasm may be early signs of excessive dosage. Other Serious Reactlons: Oscillations in performance: diurnal variations, independent oscillations in akinesia with stereotyped dyskinesias, sudden akinetic crises related to dyskinesias, akinesia paradoxica (hypotonic freezing) and 'on and off' phenomenon. Psychiatric: paranoid ideation, psychotic episodes, depression with or without development of suicidal tendencies and dementia. Rarely convulsions (causal relationship not established). Cardiac irregularities and/or palpitations, orthostatic hypotensive episodes, anorexia, nausea, vomiting and dizziness.
Other adverse reactions that may occur: Psychiatric: increased libido with serious antisocial behavior, euphoria, lethargy, sedation, stimulation, fatigue and malaise, confusion, insomnia, nightmares, hallucinations and delusions, agitation and anxiety. Neurologic: ataxia, faintness, impairment of gait, headache, increased hand tremor, akinetic episodes, "akinesia paradoxica", increase in the frequency and duration of the oscillations in performance, torticollis, trismus, tightness of the mouth, lips or tongue, oculogyric crisis, weakness, numbness, bruxism, priapism. Gastrointestinal: constipation, diarrhea, epigastric and abdominal distress and pain, flatulence; eructation, hiccups, sialorrhea; difficulty in swallowing, bitter taste, dry mouth: duodenal ulcer; gastrointestinal bleeding; burning sensation of the tongue. Cardiovascular: arrhythmias, hypotension, nonspecific ECG changes, flushing, phlebitis Hematologic: hemolytic anemia, leukopenia, agranulocytosis. Dermatologic: sweating, edema, hair loss, pallor, rash, bad odor, dark sweat. Musculoskeletal: low back pain, muscle spasm and twitching, musculoskeletal pain Respiratory: feeling of pressure in the chest. cough, hoarseness, bizarre breathing pattern, postnasal drip. Urogenital: urinary frequency, retention, incontinence, hematuria, dark urine, nocturia, and one report of interstitial nephritis. SpecialSenses: blurred vision, diplopia, dilated pupils, activation of latent Horner's syndrome. Miscellaneous: hot flashes, weight gain or loss. Abnormalities in laboratory tests reported with levodopa alone, which may occur with SINEMET *: Elevations of blood urea nitrogen, SGOT, SGPT, LDH, bilirubin, alkatine phosphatase or protein bound iodine. Occasional reduction in WBC, hemoglobin and hematocrit. Elevations of uric acid with colorimetric method. Positive Coombs tests reported both with SINEMET* and with levodopa alone, but hemolytic anemia extremely rare.

\section{DOSAGE SUMMARY}

In order to reduce the incidence of adverse reactions and achieve maximal benefit, therapy with SINEMET* must be individualized and drug administration continuously matched to the needs and tolerance of the patient. Combined therapy with SINEMET* has a narrower therapeutic range than with levodopa alone because of its greater milligram potency. Therefore, titration and adjustment of dosage should be made in small steps and recommended dosage ranges not be exceeded. Appearance of involuntary movements should be regarded as a sign of levodopa toxicity and an indication of overdosage, requiring dose reduction. Treatment should, therefore, aim at maximal benefit without dyskinesias.

Therapy in Patients not receiving Levodiopa:

initially $1 / 2$ tablet once or twice a day, increase by $1 / 2$ tablet every three days if desirable. An optimum dose of 3 to 5 tablets a day divided into 4 to 6 doses.

Therapy in Patlents receiving Levodopa:

Discontinue levodopa for at least 12 hours, then give approximately $20 \%$ of the previous levodopa dose in 4 to 6 divided doses.

FOR COMPLETE PRESCRIBING INFORMATION, PARTICULARLY DETAILS OF DOSAGE AND ADMINISTRATION, PLEASE CONSULT PRODUCT MONOGRAPH WHICH IS AVAILABLE ON REQUEST.

\section{HOW SUPPLIED}

Ca 8804-Tablets SINEMET * 250, dapple-blue oval, biconvex, scored, compressed tablets coded MSD 654 , each containing $25 \mathrm{mg}$ of carbidopa and $250 \mathrm{mg}$ of levodopa. Available in bottles of 100 .

\section{MOD $\mid \begin{aligned} & \text { MERCK } \\ & \text { SHARP }\end{aligned}$ \& DOHME canada limited}




\section{A long awaited,}

much needed and

significantly safer

preparation for

the prophylactic

treatment of

migraine

NEW
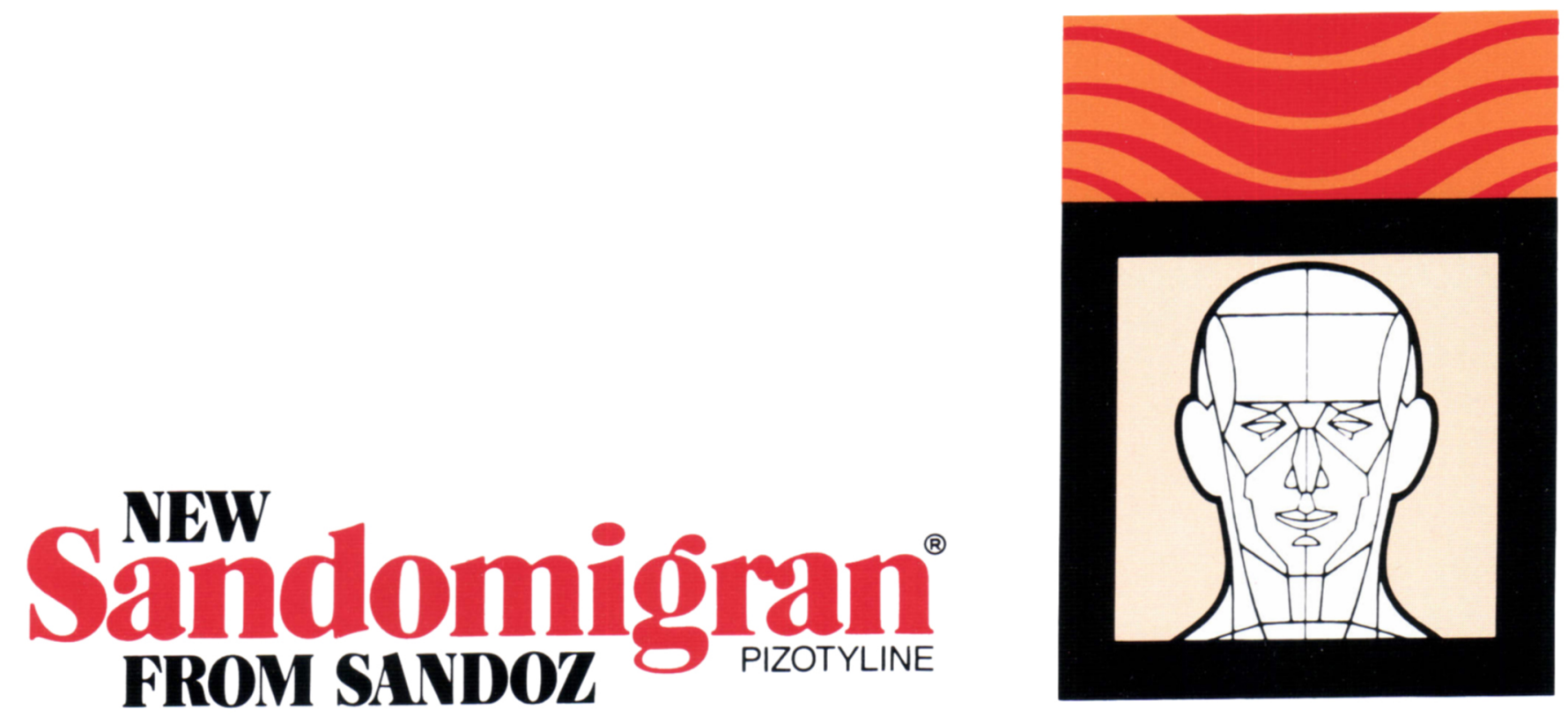


\section{Sandomigran or reduces its frequency significant}

\section{Prophylaxis of migraine: the problem.}

The prophylactic treatment of vascular headaches has been hampered sometimes by the fact that the most effective agent (methysergide) for the prevention of migraine is associated with certain undesirable side effects. Because of this, the prophylactic therapy of migraine has been confined to a relatively small, select group of patients.

\section{Overcoming the problem.}

Extensive research and wide clinical experience have shown that Sandomigran is a highly effective agent against migraine. Chemically unrelated to methysergide, Sandomigran is free of the undesirable side effects which have sometimes interfered with or precluded the prophylactic treatment of vascular headaches.

\section{The pharmacological properties of Sandomigran.}

Migraine or vascular headache is not, according to many investigators, purely of vascular origin. Many researchers believe that the biogenic amines play an important role in the pathogenesis of migraine.

Chemically unrelated to methysergide, Sandomigran (pizotyline) is a benzocycloheptathiophene derivative possessing strong antagonistic action against certain biogenic amines such as serotonin and histamine and, to a lesser degree, tryptamine, acetylcholine and the catecholamines.

Sandomigran is indicated in the prophylactic - not the symptomatic - treatment of vascular headaches.

\section{Patient selection.}

Sandomigran should be considered primarily for the more serious cases of migraine; patients who suffer two or more severe headaches every month.

Sandomigran should also be considered for patients whose headaches do not respond to symptomatic treatment.

\section{7 of every 10 migraine patients may benefit from Sandomigran.}

An analysis of 10 controlled studies ${ }^{1-10}$

(392 patients) shows the following

gratifying results:

Excellent results

(Complete disappearance of headaches)

Good results

(Reduction in frequency and

severity of headaches by at

least $50 \%$ )

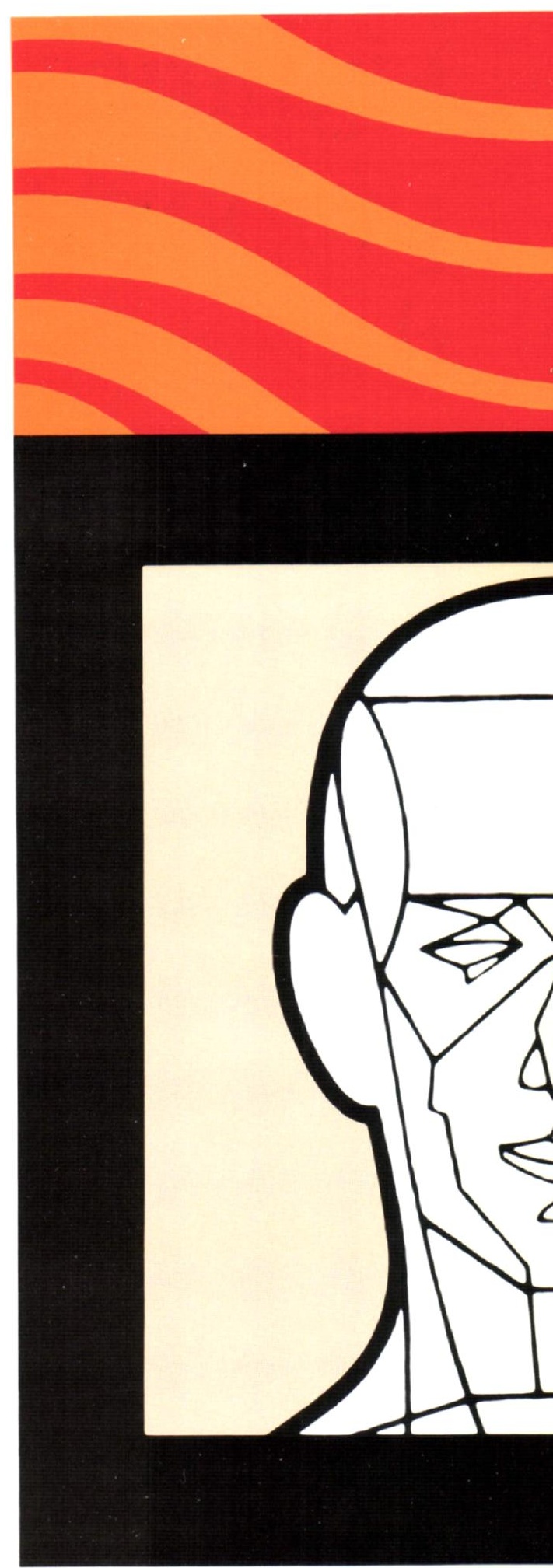




\section{Chemistry}

The chemical structure of pizotyline (Sandomigran) is totally different to the chemical structure of either methysergide (Sansert) or ergotamine.

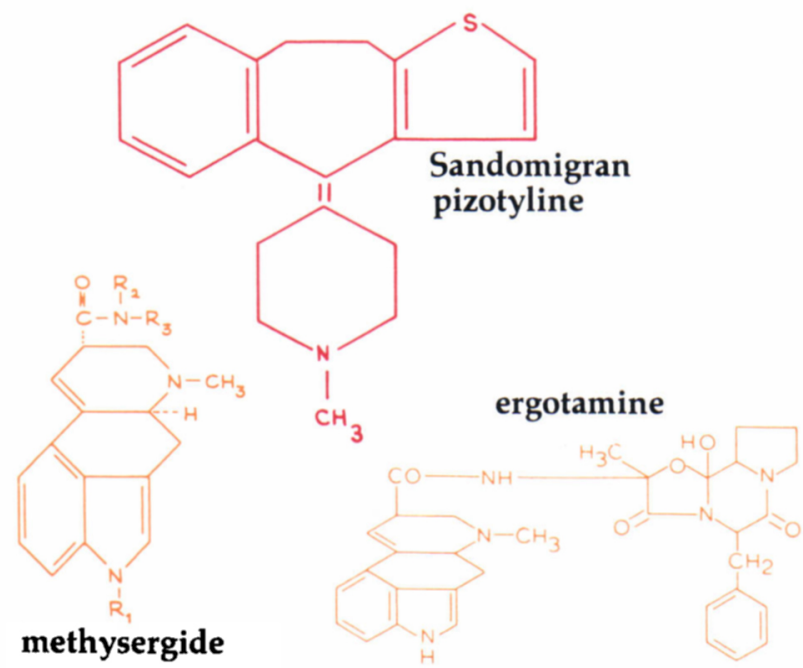

\section{Prescribing information}

Dosage - The average maintenance dosage is 1 tablet $(0.5 \mathrm{mg})$ t.i.d. A progressive dosage is recommended until the fifth day of therapy. Treatment should begin with $1(0.5 \mathrm{mg})$ tablet at bedtime (first two days), 1 tablet at noon, and at bedtime (next two days), and 1 tablet in the morning, at noon, and at bedtime (from the fifth day onward). The dosage range is 2 to 12 tablets ( 1 to $6 \mathrm{mg}$ ) per day. Since vascular headache is a paroxysmal but basically chronic disorder, treatment must extend over an adequate period of time in order to obtain maximal benefit. While some patients have responded rather quickly, most investigators agree that a fourweek trial period should be instituted to determine the true efficacy of pizotyline in specific cases. The periodic nature of the disorder will have to be considered in determining when and for how long therapy should be maintained.

Since some investigators have observed a change in headache pattern after several months of therapy, a drug-free interval is advisable to reassess the necessity of continuing treatment. The dosage should be reduced gradually during the last two weeks of each treatment course to avoid a "headache rebound."

Composition - Each ivory-coloured, sugar coated tablet contains $0.5 \mathrm{mg}$ of pizotyline as the hydrogen malate.
Side effects - Increased appetite, weight gain, and drowsiness are the most frequent side effects. An appropriate diet should be recommended by the physician for patients benefiting from the drug but gaining excessive weight. A gradual increase in the dosage of pizotyline is recommended to minimize or reduce the incidence of drowsiness.

The following adverse effects have been observed less frequently in relation to the aforementioned reactions: fatigue, nausea, dizziness, headache, confusion, edema, hypotension, depression, weakness, epigastric distress, dry mouth, nervousness, impotence and muscle pain.

Warnings and precautions - Since drowsiness may occur with pizotyline, sensitive patients should be cautioned against activities requiring rapid and precise responses (i.e. driving an automobile or operating dangerous machinery) until their response to the drug has been determined. Since the effects of antihistamines can potentiate those of other drugs affecting the central nervous system, patients should be cautioned against drinking alcoholic beverages or taking hypnotics, sedatives, psychotherapeutic agents or other drugs with CNS depressant effects during pizotyline therapy. Since it is desirable to keep drug administration to a minimum during pregnancy, pizotyline should be given only when the benefits derived from treatment exceed the possible risks to mother and fetus.

Some patients developed tolerance to pizotyline with prolonged use of the drug. An increase in dosage may overcome this tolerance.

After prolonged use hepatotoxic effects might occur and patients should be advised to report for adequate laboratory evaluation. Patients with diabetes, cardiovascular disease and known or suspected impaired renal or hepatic function should be given pizotyline with caution, and appropriate laboratory tests should be done at regular intervals.

Lens opacities occurred in two cases but did not appear to be drug-related. However, it is recommended that any impairment in vision be reported to the attending physician for further investigation.

Contraindications - Glaucoma, pyloroduodenal obstruction, stenosing pyloric ulcer and predisposition to urinary retention. Pizotyline is also contraindicated in patients taking monoamine oxidase inhibitors and for patients who have a known sensitivity to the drug. Until further studies are completed, the drug is not recommended for children under the age of twelve.

Supply - Bottles of 100 tablets.

\section{References}

1. Sicuteri, F. et al, Int. Arch. Allergy 31:78, 1967 2. Graham, J.R., Headache Rounds No. 90, 1968 3. Diemath, H.E., Arztl. Prax. 21:4994, 1969 4. Sicuteri, F. et al, Clin. ther. 40:117, 1967 5. Figueiredo, Robeiro, A., Coimbra méd. 16:8, 1969 6. Müller, E., Therapiewoche 19:1536, 1969 7. Schär, J., Praxis 57:1717, 1969 8. Pichler, E. et al, Wien. klin. Wschr. 82:208, 1970 9. Hornabrook, R.W. et al, N.Z. med. J. 70:387, 1969 10. Sercl, M. et al, Praxis 59:679, 1970

Full prescribing information is available upon request.

\section{stops migraine}

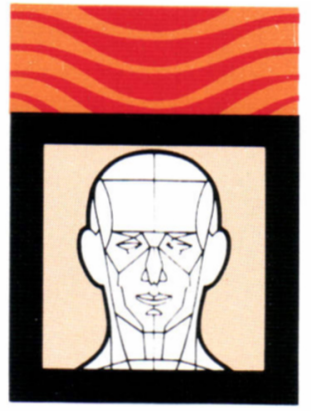

\section{before it attacks}

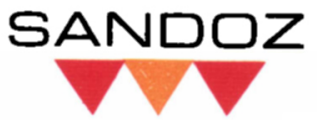




\section{田 \\ Symmetrel (amantadine $\mathrm{HCl}$ ) \\ for the management of Parkinson's syndrome}

* Chemically distinct

(Not related to levodopa or anticholinergic
* Fast onset of action

(Either initiated concurrently or added to levodopa. Additional benefit may result - such as smoothing out of fluctuations in performance which sometimes occur when levodopa is administered alone. When the levodopa dose must be reduced because of side effects, the addition of Symmetrel may result in better control of Parkinson's syndrome than is possible with levodopa alone.)

\section{Effective with other anticholinergic antiparkinson drugs}

When these drugs, e.g benztropine mesylate, provide only marginal benefits, Symmetrel used concomitantly may provide the same degree of control of Parkinson's syndrome, often with a lower dose of anticholinergic medication and a possible reduction in anticholinergic side effects.)

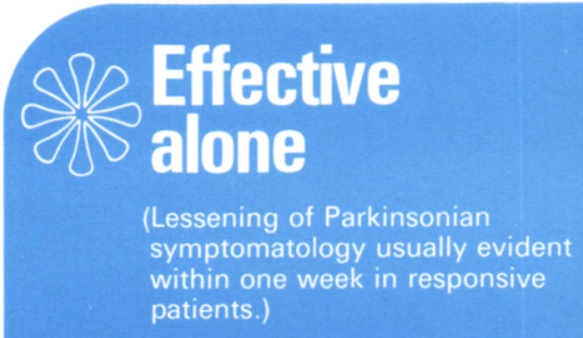
patients.)
CONTRAINDICATIONS

WARNINGS Patients with a history of epilepsy or other "seizures" should be

Patients with a history of congestive heart tailure or peripheral ederma should be receiving "Symmetrel" (amantadine $\mathrm{HCll}$

Satety of use in pregnancy has not been established Theretore. "Symmetrel" should not be used in women with childbearing potential. unless in the opinion of the phys:-

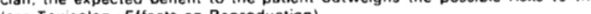
see roncolosi

Since the drug is secreted in the milk. "Symmetrel" should not be administered to

PRECAUTIONS The dose of "Symmetrel" may need careful adjustment in patients with renal impairment, congestive heart tailure, peripheral edema. or ortho. static hyootension. Since "Symmetrel" is not metabolized and is
the urine, it may accumulate when renal tunction is inadequate.

Care should be exercised when administering "S Symmetrel" io patients with liver disease, a history of recurrent ecrematord rash. or to patients with psychosis or tion is required when "Symmetrel" is administered concurrently with central nervous system stimulants

Patients with Parkinson's syndrome improving on "Symmetrel" should resume normal activities gradually and cautiously. consistent with other medical considera tions. such as the presence of osteoporosis or phiebolinrombosis

Patients receiving "Symmetrel" (amantadine $\mathrm{HCl}$ )) who note central nervous system effecis of bluring ar vision should be ca

"Symmetrel" (amantadine HCl) should not be discontinued abruptly since a few The dose of anticholinergic drugs or of " $5 \mathrm{ymm}$

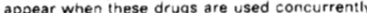

ADVERSE REACTIONS Adverse reactions reported below have occurred with anticholinergic antiparkinson drugs and/or levodopa atic hypotensive eoisodes. con gestive heart fallure. depression. Dsychosis and urinary retention. and rarely cont. Other adverse reactions of less importance which have been observed are. anorexio.

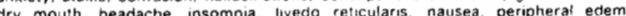
drowsiness, dysprea fatigue hyperkinesia irrilability, nightmares. rash slurred speech, visual disturbance. vomiting and weakness, and very rarely eczematord dermatitis and oculogyric episodes

of the drug

DOSAGE AND ADMINISTRATION The initial dose of "Symmetrel" is $100 \mathrm{mg}$ daily for patien/s with serious associated medical illnesses or who are receiving high doses of other antiparkinson drugs. After one to several weeks al $100 \mathrm{mg}$ once daily, the dose may be increased to $100 \mathrm{mg}$ twice daly. When "Svm melrel and levodopa are initiated concurrently. Symmetrel shous be held consian at $100 \mathrm{mg}$ daily or twice daily while the daily dose of levodopa is gradually increase a da

Patients whose responses are not optimal with "Symmetrel" (amantadine HCI) a Poo $\mathrm{mg}$ daily may benefit from an increase to $300 \mathrm{mg}$ daily in divided doses Patien who experience a fall-off of effectiveness mav regain benefit by increasing the
to $300 \mathrm{mg}$ daily. such patients should be supervised closely by their physicians DOSAGE FORMS CAPSULES. (bottles of 100) - each red. soft gelatin

Product monograoh, with complete references, available upon request

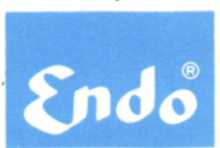

MEMEer

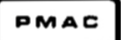

LABORATORIES MONTREAL

(1)PDN

Subsidiary of E.I. du Pont de Nemours \& Co. (Inc.) 


\section{In epilepsy*}

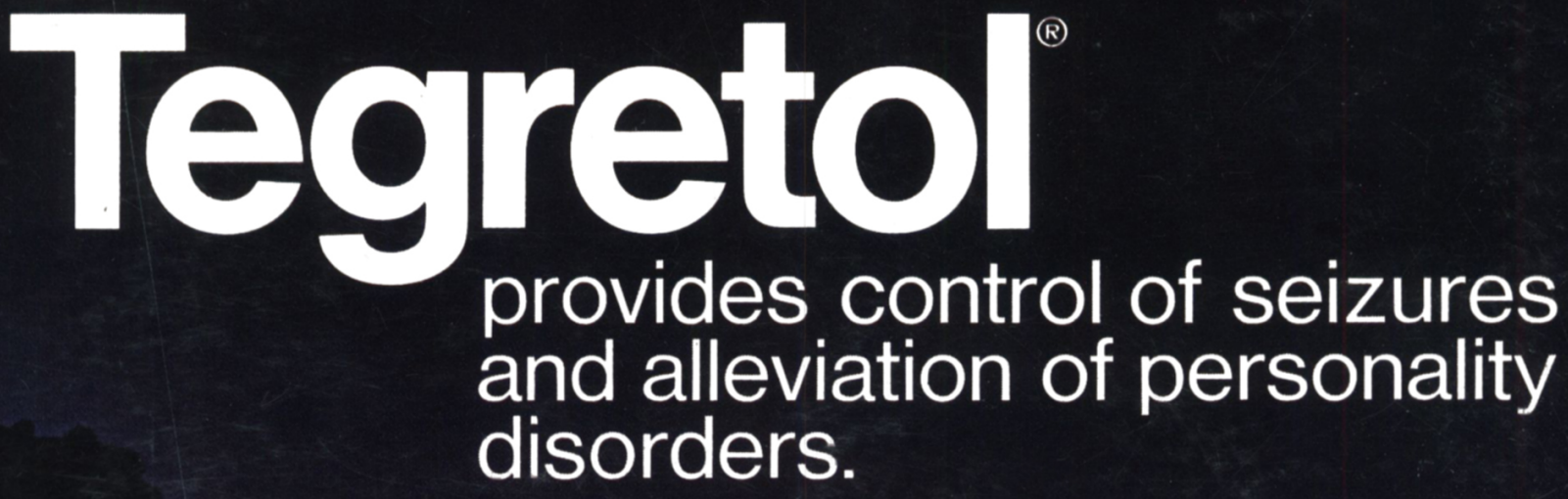

The drug of choice for patients with psychomotor (Temporal Lobe) Epilepsy

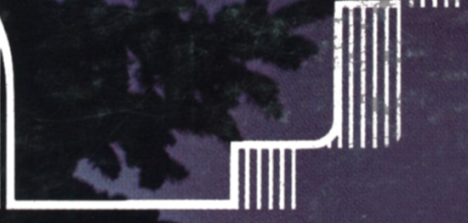

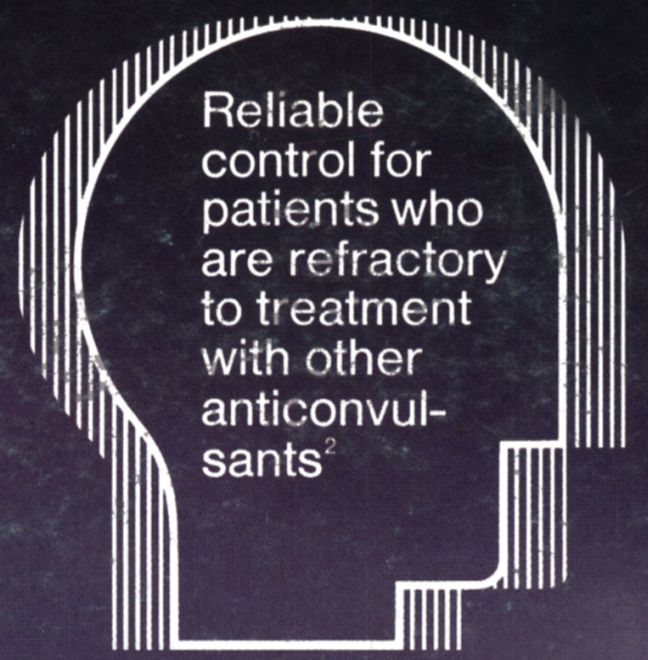
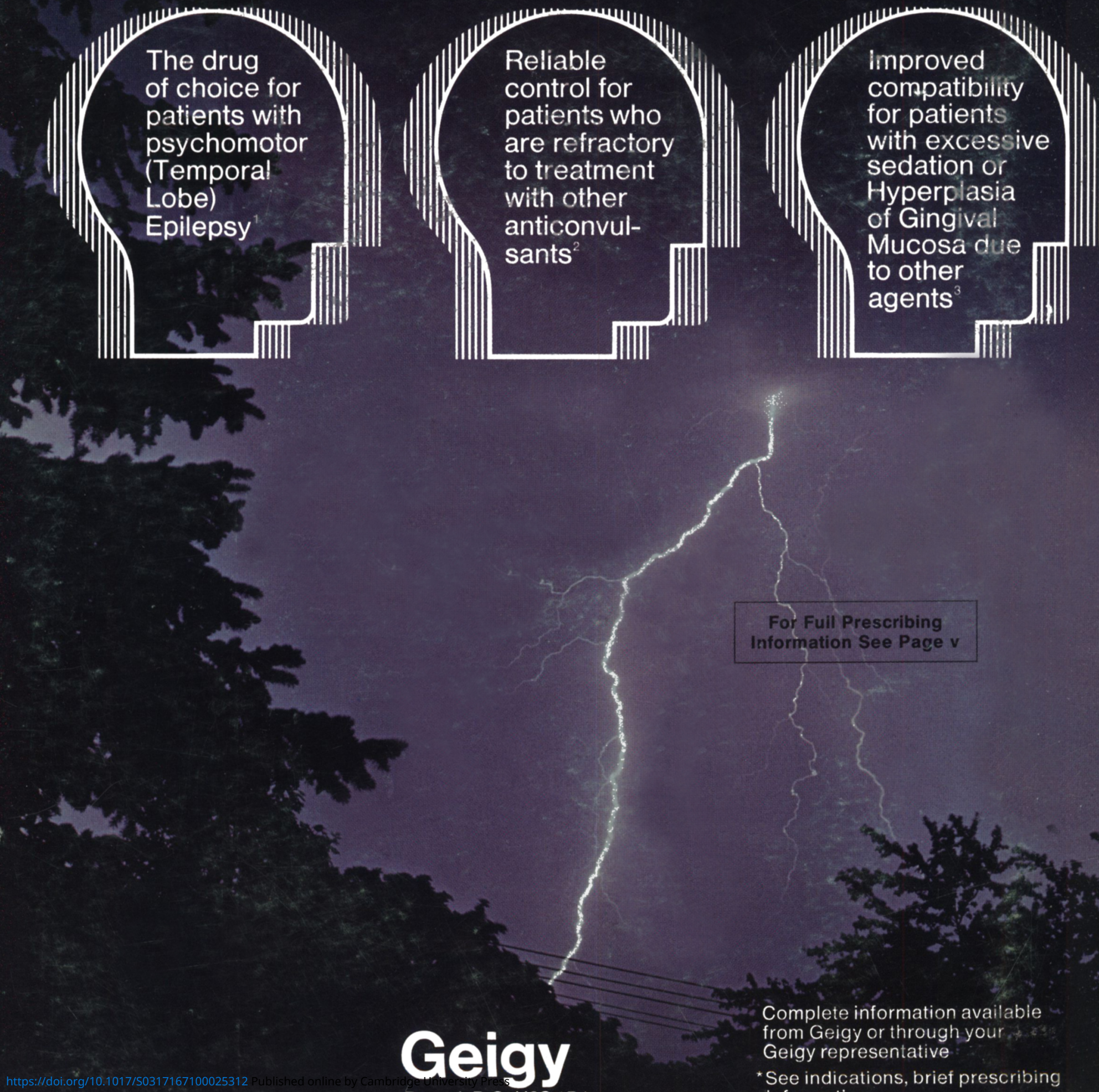

Complete information available from Geigy or through your Geigy representative

*See indications, brief prescribing information 